\title{
Controlling the Gaze Direction of a Humanoid Robot with Redundant Joints
}

\author{
Felix Faber, Maren Bennewitz, and Sven Behnke \\ Computer Science Institute \\ University of Freiburg, Germany \\ \{ faber $\mid$ maren | behnke\}@informatik.uni-freiburg.de
}

\begin{abstract}
Due to their high number of joints, humanoid robots typically have kinematic redundancies to achieve endeffector poses. Examples for such redundancies are the kinematic chains of pitch and yaw joints that allow the robot to turn towards a gaze target. Our humanoid communication robot currently uses its spine, its neck, and its eye joints to direct its cameras towards an object. In this paper, we propose a control strategy that considers three factors, namely tracking error, discomfort, defined at the joint level, and "effort" to control the pitch and yaw joints. Our strategy is based on gradient descent on a cost function. During the optimization, we use different step sizes to reflect the different inertia of the moved parts. Our control scheme produces human-like motions, where smaller, light-weight parts such as the eyes of the robot move quickly towards the target and then move back while the larger joints turn towards the target. We present experiments to evaluate the proposed strategy qualitatively and quantitatively.
\end{abstract}

\section{INTRODUCTION}

Humanoid robots have become a popular research tool in recent years. More and more research groups worldwide develop complex machines with a human-like body plan and human-like senses [1]-[4].

One of the most important motivations for humanoid robot research is that such robots are suitable for intuitive multimodal communication with humans. Our communication robot interacts with multiple persons in its vicinity using speech, gestures, facial expressions, and body language. It detects and tracks multiple persons using audio-visual data. The robot uses multiple modalities to decide which person gets its attention. While focusing on one person, the robot still involves other people into the interaction by establishing short eye-contact or reacting to speech [5].

Because they mimic the kinematic structure of humans, humanoid robots are equipped with a high number of joints. Typically, these joints create kinematic redundancy. Examples for such redundancies are the kinematic chains of pitch and yaw joints that allow the robot to turn towards a gaze target. Our robot currently uses its spine, its neck and its eye joints to direct its cameras towards an object. Later, we plan to consider also the legs for turning.

Such redundancies create room for pursuing multiple goals in controller design. The goals that are relevant for our application can be grouped into machine-oriented goals and human-oriented goals. The machine-oriented goals include the joint limits, a low tracking error of the target to be tracked, and a low control effort. They are relatively easy to quantify, based on the readings of robot sensors. In contrast to that, the human-oriented goals are more difficult to specify. Here, aspects like the resemblance of the motion to human motion or the perceived naturalness of the produced movements are desired.

Fortunately, machine-oriented and human-oriented goals are not contradictory. We show that optimizing machineoriented goals in humanoid robots can lead to natural, human-like motions.

In this paper, we present an approach to control the redundant kinematic chains of pitch and yaw joints between the floor and the robot cameras that takes into account 1) the tracking error regarding the person or the object that has to be focused, 2) the inertial tensor of the moved parts, and 3) the "discomfort" or "tension" of individual joints. Based on these factors, we define a cost function and compute trajectories for the individual joints so that the costs are minimized. During the optimization, we avoid high computational demands by applying a simplification that is justified for our application.

In the experimental section, we evaluate our approach qualitatively and quantitatively and compare it to other control strategies. The experiments show that our controller produces human-like motions, where smaller, light-weight parts move quickly towards the target and then move back while the larger joints turn towards the target.

The remainder of this paper is organized as follows. After the discussion of related work in the next section, we cover the design of our humanoid communication robot and its attentional system in Sections III and IV, respectively. In Section $\mathrm{V}$ we detail the derivation of our controller. In Section VI we present experiments illustrating the characteristics of our control strategy. Furthermore, we compare our approach to different other control strategies.

\section{RELATED WORK}

\section{A. Interaction}

Several systems exist that use data provided by different sensors to detect and track people during an interaction. Various strategies are then used to decide which person gets the attention of the robot. For example, the system developed by Spexard et al. [3] uses data provided by two cameras and two microphones to detect and track people. The authors consider the person that is currently speaking as the person of interest. Okuno et al. [6] follow a similar strategy. They apply two different modes. In the first mode, the robot always 
turns to a new speaker, and in the second mode, the robot keeps its attention exclusively on one conversational partner. Tasaki et al. [7] define a "friendliness space" given audiovisual data to decide which person gets the focus of attention. Blanco et al. [8] presented an approach to track users with a combination of laser range and visual data. The pan and tilt of the robot's camera are adjusted so as to focus the closest person.

Furthermore, several research groups study human-robot interaction mainly focusing on scenarios with a single person. For example, Stiefelhagen et al. [4], Bischoff and Graefe [9], and Breazeal et al. [1] have been working on natural interaction between humans and a robot using multiple modalities such as speech and gestures. Böhme et al. [10] proposed to adapt the robot's behavior according to the user's age, gender, and mood. Sidner et al. [11] analyze how the attention of humans can be drawn to specific objects of interest. Aoyama and Shimomura [12] developed a framework for real-world speech interaction with a humanoid robot. Salichs et al. [13] presented a robotic platform that is able to cooperatively dance with human partners.

All the above mentioned systems move the joints of the robot in order to turn towards a person or toward an object of interest. However, it is not specified in which way individual joints, e.g., the upper body, the neck, and the eyes, are moved exactly.

Matsui et al. [2] try to generate natural, human-like motion using a motion capture system. The similarity between the motion of a human and the movements of an android is measured by differences of the motion at the visible surface.

\section{B. Multiple Objectives Control}

We propose to control the redundant yaw and pitch joints in a kinematic chain by applying gradient descent using an cost function that takes into account 1) the tracking error regarding the person or the object that has to be focused, 2) the mass that has to be moved, and 3) the "discomfort" or "tension" of individual joints. Several concepts of such an approach have been presented before.

Our approach is related to $\mathrm{Gu}$ and Ballard [14] that apply the Equilibrium-point hypothesis [15] to control joint movements. They proposed a two-phase control model. First, gradient descent is applied to obtain joint target angles given a Cartesian end-effector target. Second, muscles are simulated using springs that drive the execution of the movements. $\mathrm{Gu}$ and Ballard define an energy function that also considers the mass and the difference between the current and target joint configuration (which is the tracking error in our case). The discomfort factor of the joints is implicitly modeled by the simulated muscles. Since in our application scenario, the configuration of the joint angles has possibly to be changed every time step according to moving targets that have to be tracked, we apply gradient descent at every time step and do not apply a two-phase model.

Kathib [16] introduced an artificial potential field approach for real-time obstacle avoidance for manipulators and mobile robots. In his work, he also used an approach to satisfy the
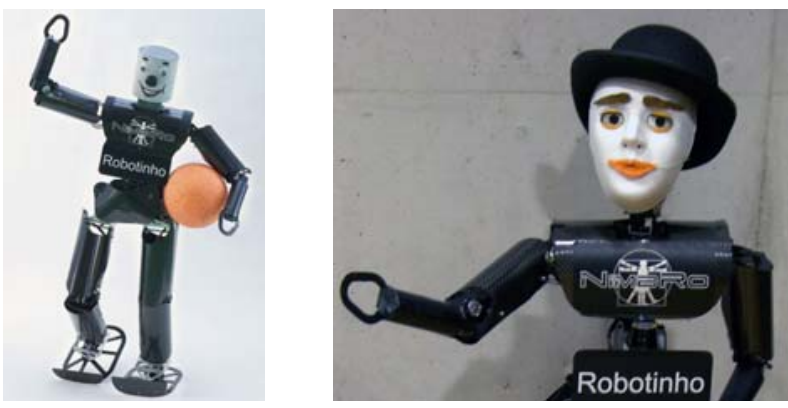

Fig. 1. Our robot Robotinho was initially used as soccer player in the RoboCup Humanoid League. Recently, the robot was equipped with a 2DOF neck and a $15 \mathrm{DOF}$ communication head.

manipulator internal joint constraints. The joint positions are kept within the joint limits by creating barriers of potential at each of the limits. He proposed a partial linearization of the potential function, to avoid a drift towards the middle of the interval. As Kathibs approach works in configuration space it is, compared to our approach, computationally very demanding.

Marchand et al. [17] introduced a task function that combines the execution of vision-based tasks while minimizing a secondary cost function. In the cost function, joint positions close to limits yield higher costs. The partially linearized cost function is minimized by following the gradient in the negative direction.

Nakamura et al. [18] utilize the redundancy in kinematic chains to perform lower priority tasks, such as collision avoidance, while performing high priority tasks, such as tracing a given trajectory with an endefector. The integration of prioritized tasks for redundant manipulators has recently gained new interest for humanoid robots like for example in Mansard et al. [19] or Sugiura et al. [20]. Our approach also considers different tasks but does not require any prioritization between them.

Optimizing multiple goals simultaneously is generally common in biological systems. Todorow compiled a review of optimal control in sensorimotor systems [21].

Finally, it should be noted that various studies have been carried out to investigate discomfort in human movement without incorporating them in an optimization-based performance measure [22]-[24]. Marler [25] modeled human discomfort and included it in a multi-objective optimization for posture prediction for virtual humans.

\section{DESIGN OF OUR Robot}

Our humanoid robot Robotinho, shown in Fig. 1, has been originally designed for playing soccer in the RoboCup Humanoid League [26]. Robotinho is $110 \mathrm{~cm}$ tall and has a total weight of about $6 \mathrm{~kg}$. Its body has 25 degrees of freedom (DOF): six per leg, four per arm, three in the trunk, and two in the neck. The mechanical design for the body focused on simplicity, robustness, and weight reduction. The body is driven by $37 \mathrm{DX}-117$ actuators.

Robotinho's skeleton is constructed from aluminum extrusions with rectangular tube cross section. In order to reduce 
weight, we removed all material not necessary for stability. The feet and the forearms are made from sheets of carbon composite material. The robot is protected by a layer of foam and an outer shell of thin carbon composite material.

For the use as communication robot, we equipped Robotinho with an expressive 15DOF head, shown in the right part of Fig. 1. All joints are driven by small digital servos. The eyes are movable USB cameras. Four servos move the eyes in two axis. While the lower eye lid moves together with the eyeballs, the upper eye lid can be moved independently in pitch direction. Six servo motors animate jaw and mouth. One pair of servos moves the jaw in pitch direction. Each mouth corner is moved by two servos. Four servos animate the two eyebrows.

Robotinho is equipped with a CardS12 and two ChipS12 microcontroller boards, which manage the communication with the actuators. Every $12 \mathrm{~ms}$, target positions and compliances for the actuators are sent from the main computer to the microcontroller board, which distribute them to the actuators. Sensor readings are sent back to the main computer.

We use a tiny Sony Vaio UX PC as main computer. The weight of the UX is only $0.5 \mathrm{~kg}$. It is attached to the lower back of Robotinho. A mini-USB hub connects the two cameras (Videology $21 \mathrm{~K} 155$ ) to the main computer. The cameras are small $(22 \times 26 \mathrm{~mm})$ high-resolution $(765 \times 582$ pixels) color cameras with high-speed USB 2.0 interface. One camera has a narrow field-of-view of $21.4^{\circ}$ while the other camera is equipped with a wide-angle lens, yielding a horizontal field-of-view of $64^{\circ}$. Additionally, the robot is equipped with a stereo microphone.

Robotinho is fully autonomous. The robot is powered by high-current Lithium-polymer rechargeable batteries, which are located in its hip.

\section{Attentional System}

Our robot is able to detect and track multiple persons using audio-visual data. In order to keep track of people even when they are temporarily outside the robot's field of view, the robot maintains a probabilistic belief about the people in its surroundings.

It is not human-like to fixate a single conversational partner all the time when there are other people around. Therefore, our robot uses multiple modalities to decide which person currently gets its attention. The robot shows interest in different persons in its vicinity and shifts the attention between them so that they feel involved into the conversation [5].

Our robot uses arm and head movements to generate gestures that support the speech synthesis as well as gestures with its animated face. For example, to draw the attention of communication partners towards objects of interest, our robot performs pointing gestures. When the robot wants to draw the attention to an object, it simultaneously turns itself towards the object and points in its direction with the respective arm while uttering the object name.

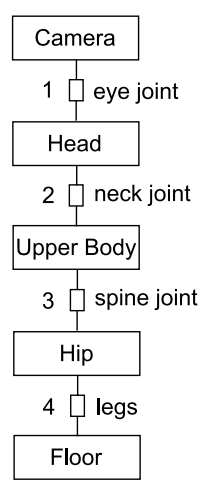

Fig. 2. Four redundant joints that form a kinematic chain can be used to turn the robot cameras towards a target in yaw direction.

\section{Gaze And Movement Control}

During interaction, our robot has to track objects, such as faces, that it localizes in the camera images. We define the angular tracking errors $\theta_{\text {target }_{p}}$ and $\theta_{\text {target }_{y}}$ as the angles between the center of the camera images and the object of interest for the pitch and yaw direction. Tracked objects outside the current field of view are kept in a global coordinate system.

For turning the robot towards a target, we use four pitch and four yaw joints that form kinematic chains for the pitch and yaw direction: The pitch and yaw eye joints to turn the cameras, the pitch and yaw neck joints to turn the head, the pitch and yaw spine joints to turn its upper body, and finally the legs, for turning the whole body. As our current application scenario (i.e., explaining exhibits which are placed in front of the robot) is stationary, the legs are currently not used to turn the robot. Turning towards a gaze target during omnidirectional walking has been used for soccer and will be used again soon when we extend the application scenario to a mobile museum guide. Fig. 2 illustrates the redundant kinematic chain for the yaw direction.

Each of these joints of one direction (pitch or yaw) can be used to minimize the corresponding $\theta_{\text {target }}$ by simply turning in the direction of it. Due to the kinematic redundancy, however, it is not clear which joint or combination of joints should be used. One could use only one joint to turn the whole way or multiple joints and divide the tracking error between them.

Besides the tracking error $\theta_{\text {target }}$, which has to be minimized, we have to consider the physical limits of each joint. Running against these physical limits must be avoided at all times in order not to damage the joint. Joint positions close to physical limits are also not favorable. In these positions, the joint is not able to turn further and tracking error compensation relies completely on other, possibly energy inefficient joints. Furthermore, the robot's posture might look unnatural in such an extreme position. In order to describe unfavorable joint positions, we introduce the notion of discomfort. The discomfort function $U$ of a joint position $\alpha_{k}$ yields a high value if $\alpha_{k}$ is close to a physical limit. When the joint position is far away from the limits, the 


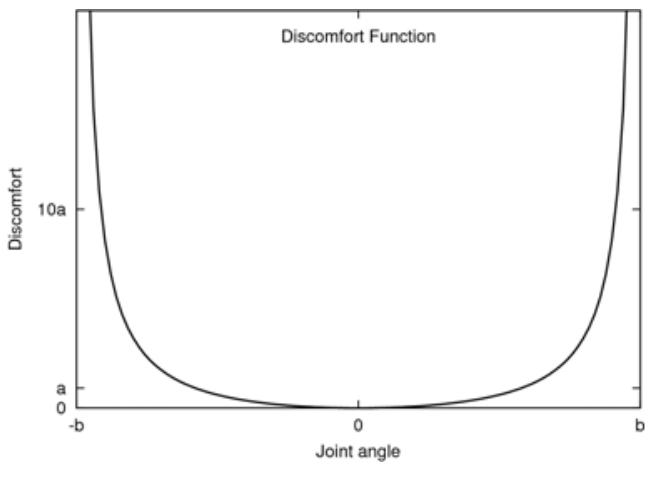

Fig. 3. Discomfort function $U(\alpha)$ for $a=10$ and $b=1$. See Equation 1.

discomfort of a joint is small. The least discomfortable joint position is reached when $\alpha_{k}=0$, where $U(0)=0$. We define $U$ as the summation of two hyperbolic functions (Fig. 3). $U$ is characterized by the shaping factor $a$ and the poles, corresponding to the physical joint limits, at $\pm b$

$$
U\left(\alpha_{k}\right)=\frac{a}{\alpha_{k}+b}+\frac{a}{-\alpha_{k}+b}-\frac{2 a}{b} .
$$

For technical reasons, we linearize $U$ at and beyond the poles. Our goal is to minimize the discomfort $U\left(\alpha_{k}\right)$ for each joint $k$.

To determine how far and in which direction the joints have to move at the next time step, we formulate a cost function $E$ that is based on the two goals, minimizing the tracking error $\theta_{\text {target }}$ and the discomfort $U$ :

$$
\begin{aligned}
& E\left(\alpha_{1}^{t+1}, \ldots, \alpha_{K}^{t+1}\right)= \\
& \quad \sum_{k=1}^{K} \frac{1}{2} U\left(\alpha_{k}^{t+1}\right)^{2}+\frac{1}{2}\left[\theta_{\text {target }}-\sum_{k=1}^{K}\left(\alpha_{k}^{t+1}-\alpha_{k}^{t}\right)\right]^{2}
\end{aligned}
$$

where $\alpha_{k}^{t+1}-\alpha_{k}^{t}$ is the amount of change in joint position of joint $\alpha_{k}$ between timestep $t$ and $t+1$.

We then calculate the gradient of the cost function at the current joint position for each joint. In order to do so, we compute the partial derivative for each joint position $\alpha_{k}^{t+1}$

$$
\frac{\partial E}{\partial \alpha_{k}^{t+1}}\left(\alpha_{k}^{t}\right)=\left[\frac{\partial U}{\partial \alpha}\left(\alpha_{k}^{t}\right) \cdot U\left(\alpha_{k}^{t}\right)-\theta_{\text {target }}\right]
$$

and get the gradient

$$
\nabla E_{\left(\alpha_{1}^{t+1}, \ldots, \alpha_{K}^{t+1}\right)}=\left[\frac{\partial E}{\partial \alpha_{1}^{t+1}}\left(\alpha_{1}^{t}\right), \ldots, \frac{\partial E}{\partial \alpha_{K}^{t+1}}\left(\alpha_{K}^{t}\right)\right] .
$$

We apply the gradient descent method for reaching a minimum of $E$. We use a step size $\eta$ and follow in the opposite direction of the gradient with each joint.

The further down the $k$ th joint is located in the kinematic chain, the greater is the moment of inertia of the masses that are moved by it. For example, while the eye joint only has to turn the small weight of the cameras, the spine joint already has to turn the whole upper body. Certainly, greater efforts by a joint are implied at greater moments of inertia. Since the aim should be to save resources, our goal is to limit the

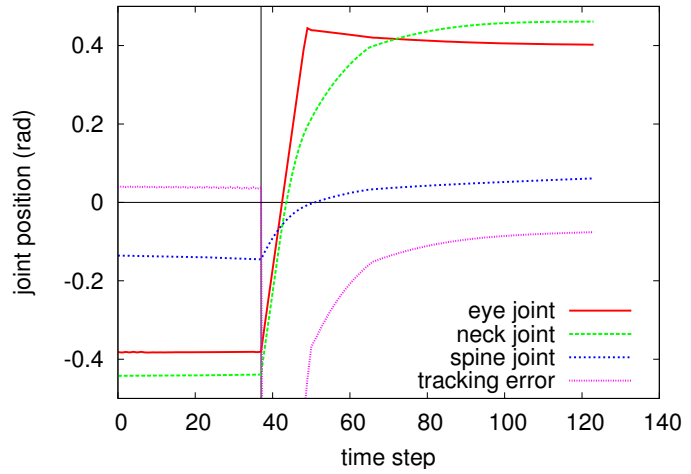

Fig. 4. Response of the robot to an instantaneous position change of the tracking target.

effort of all joints when turning. As a result, we scale the size of the steps with the inverse moment of inertia $I_{k}$ of each joint. This can also be motivated by a linear quadratic regulator (LQR) [27], which balances tracking error and control effort. The LQR also uses a lower feedback gain when the control effort for a position change is higher. In effect, the tracking error is reduced at different time scales for the different joints.

Finally, we take into account that the joints have a maximum angular velocity $v_{\max }$ that cannot be exceeded. This leaves us with the update rule

$$
\alpha_{k}^{t+1}=\alpha_{k}^{t}+\operatorname{sat}\left(v_{\max },-\frac{\partial E}{\partial \alpha_{k}^{t+1}}\left(\alpha_{k}^{t}\right) \cdot \frac{\eta}{I_{k}}\right),
$$

where $\operatorname{sat}\left(v_{\max }, x\right)$ saturates $x$ at $\pm v_{\max }$.

\section{EXPERIMENTAL EVALUATION}

\section{A. Qualitative Evaluation of Our Control Strategy}

We tested our control strategy on the robot in different settings. In all experiments, the shaping factor $a$ of the discomfort function $U$ was set to 0.1. As a result, the discomfort function is almost linear in the center of the interval. This follows the idea that human joint positions close to the center of the interval do not cause relevant discomfort. The physical joint limits $b$ are $\pm 0.5 \mathrm{rad}$ for the eyes, $\pm 0.56 \mathrm{rad}$ for the neck and $\pm 1.11 \mathrm{rad}$ for the spine, respectively. The step size $\eta$ was set to 0.05 . The experiments were conducted in the yaw-plane, using yaw joints only as the absolute angular range which can be covered is largest in this plane.

As a gaze target, we placed a virtual object at different angles relative to the static feet in front of the robot. In a first trial (Fig. 4), the robot was looking at a target at $-1.0 \mathrm{rad}$. The robot had been looking at this target for quite some time and thus all joints had reached a steady state. At time step 37 the target instantaneously changed its position and was then located at an angle of $1.0 \mathrm{rad}$. As a consequence, the eye joint rapidly turned until the position get too uncomfortable in time step 43. The neck joint, which has to move a greater mass, followed slightly slower and allowed the eyes to turn back from their current uncomfortable position. The spine joint has to move by far the largest mass and thus showed the slowest response. This corresponds to our observation of 


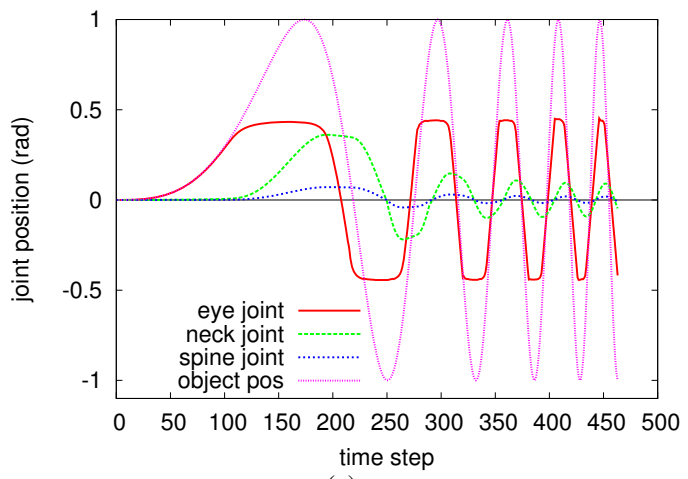

(a)

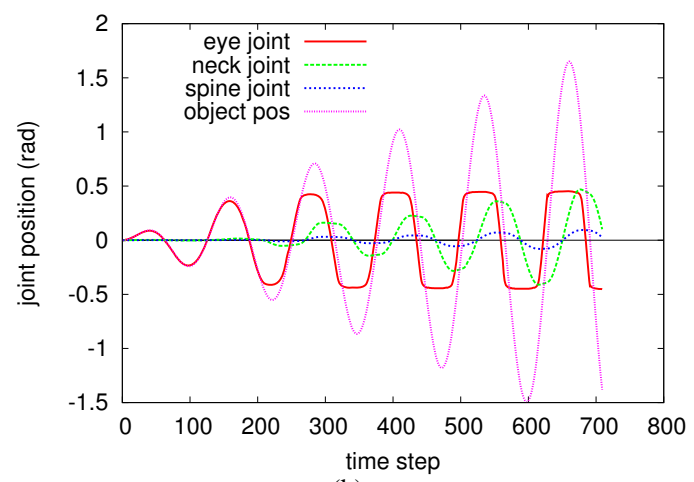

(b)

Fig. 5. Tracking a target that oscillates in front of the robot. In (a) the frequency of the oscillation is increased over time while in (b) the frequency stays constant but the amplitude is increased.

human motion. For a human, the eyes are also the part of the body that can move fastest and with increasing discomfort accelerate neck and spine movement. When humans track a static target, they also turn towards the target with more body parts after fixating it for some time.

In a second experiment, we let an object oscillate with increasing frequency in front of the robot (Fig. 5(a)). One can clearly see how the fractions of the tracking error compensation changed as the frequency rises. In the beginning, especially the neck had a great fraction on the compensation of the tracking error. This fraction decreases with increasing frequency. The spine showed a similar behavior. Note that for tracking fast, oscillating objects, humans also mainly use the eyes while for slow moving objects other body parts are utilized, too.

Furthermore, we performed a third experiments in which again an object oscillated in front of the robot, but this time with increasing amplitude (Fig. 5(b)). Here it is very obvious, that when the eye joint reaches uncomfortable positions close to the joint limits, the neck joint is forced to move and also the spine joint. In human motion, the discomfort caused by eye positions also leads to neck and spine movements.

\section{B. Comparison with Different Control Strategies}

In further experiments, we compared strategies for turning towards a target using all or some of the available joints. At the beginning of each experiment, we let the robot look to a neutral, comfortable position straight forward. We then placed two objects at a certain angle on each side of the robot. The robot's task was now to look alternatingly every four seconds from one object to the other. The trial finishes after the robot had changed targets five times. We repeat the trial for different object angles. With this experimental setup, we explore how the different strategies perform on executing fast saccades as well as on tracking a static object.

During these experiments, the total amount of discomfort present in the system, the amount of work needed, and the integrated tracking error was recorded. The resulting values of different strategies were then compared. Joint limits, masses and maximum joint speeds were fixed. When calculating the performed work, we are not so much interested in the exact amount of work performed, but rather in the different proportions of the bodyparts to each other. Thus, we approximated the inertia of the body parts according to their mass and radius proportions. We treated the eyes and the head as a sphere and the trunk as a cylinder.

Apart from testing our control strategy with all available joints, we also test it with a disabled spine joint and after that with disabled spine and neck joints. We then compare our strategy to one that does not take discomfort and mass distributions into account. In this simple strategy, each joint tries to minimize the tracking error by itself simultaneously. Results of the trials are depicted in Figure 6.

The experiments show that using additional joints leads to less overall discomfort and a smaller tracking error. Furthermore, it can be seen that a strategy that does not take into account discomfort and masses is less energy efficient and causes large discomfort in the joints.

\section{CONCLUSION}

In this paper, we suggested a control strategy for the redundant kinematic chains of pitch and yaw joints between the floor and the cameras of our humanoid communication robot. This strategy balances tracking error, joint-limit avoidance, and control effort. It is implemented by a state-feedback control law at different levels of the kinematic chain. The approach is a simplification of previous works in the field of multi-objective optimization for motion control, applied to a real robotic system.

The experiments show that the proposed strategy produces trajectories that qualitatively resemble human motion. In particular, we observed in saccades that the cameras are moved quickly towards the target, but that the discomfort caused by eye positions close to the joint limit leads to neck and spine movements, which allows the eye joint to come back to a more comfortable position.

In the steady state, tracking error is balanced against discomfort. For targets close to the center of the floor coordinate system, discomfort is minimal and the target is reached almost perfectly. In contrast to that, for targets far away from the center, the discomfort is reduced by accepting a larger tracking error in the steady state.

The experiments demonstrated that quick oscillatory movements are tracked predominantly with the eyes, while 
(a)

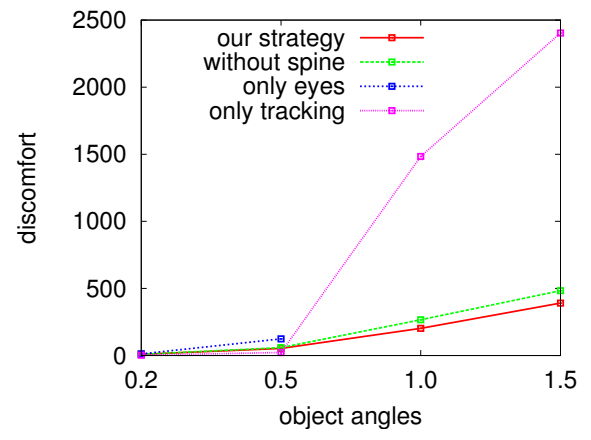

(b)
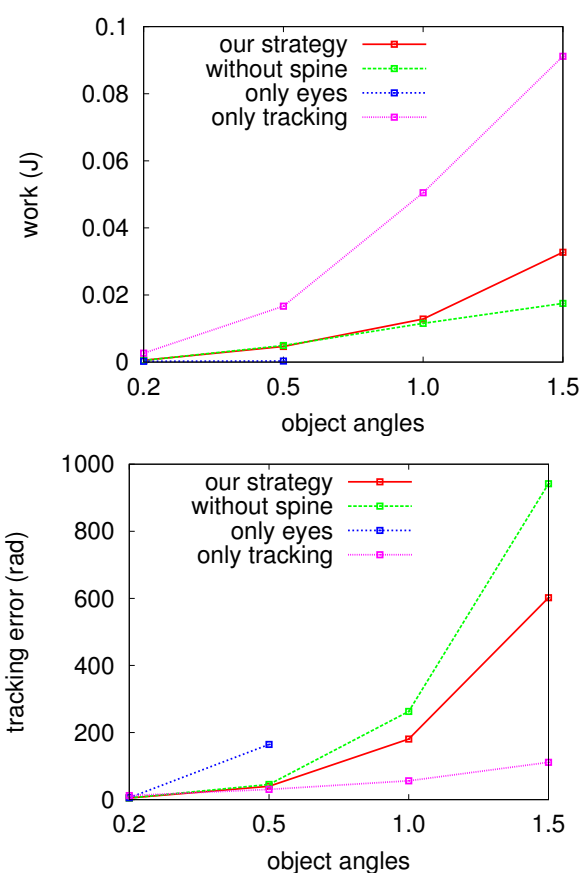

Fig. 6. The (a) integrated discomfort, (b) performed work, and (c) integrated tracking error at an object angle of 0.2, 0.5, 1.0 and $1.5 \mathrm{rad}$ for the compared strategies. See text for details.

for following slower target motions, the lower joints in the chain contribute more. When observing our humanoid robot, the generated motions appear natural and human-like. Due to the combined range-of-motion of the pitch and yaw joints, the robot is able to look at objects far-away from the center of the floor coordinate system.

Future work will include the quantitative comparison of our control strategy with human data.

\section{REFERENCES}

[1] C. Breazeal, A. Brooks, J. Gray, G. Hoffman, C. Kidd, H. Lee, J. Lieberman, A. Lockerd, and D. Mulanda, "Humanoid robots as cooperative partners for people," Int. Journal of Humanoid Robots, vol. 1, no. 2, pp. 1-34, 2004.

[2] D. Matsui, T. Minato, K. F. MacDorman, and H. Ishiguro, "Generating natural motion in an android by mapping human motion," in Proc. of the IEEE/RSJ Int. Conf. on Intelligent Robots and Systems (IROS), 2005.

[3] T. Spexard, M. Hanheide, and G. Sagerer, "Human-oriented interaction with an anthropomorphic robot," IEEE Trans. on Robotics, vol. 23, no. 5, pp. 852-862, 2007.

[4] R. Stiefelhagen, H. Ekenel, C. Fügen, P. Gieselmann, H. Holzapfel, F. Kraft, K. Nickel, M. Voit, and A. Waibel, "Enabling multimodal human-robot interaction for the Karlsruhe humanoid robot," IEEE Trans. on Robotics, vol. 23, no. 5, pp. 840-851, 2007.
[5] M. Bennewitz, F. Faber, D. Joho, S. Schreiber, and S. Behnke, "Towards a humanoid museum guide robot that interacts with multiple persons," in Proc. of the IEEE-RAS Int. Conf. on Humanoid Robots (Humanoids), 2005.

[6] H. Okuno, K. Nakadai, and H. Kitano, "Social interaction of humanoid robot based on audio-visual tracking," in Proc. of the Int. Conf. on Industrial and Engineering Applications of Artificial Intelligence and Expert Systems (IEA/AIE), 2002.

[7] T. Tasaki, K. Komatani, T. Ogata, and H. Okuno, "Spatially mapping of friendliness for human-robot interaction," in Proc. of the IEEE/RSJ Int. Conf. on Intelligent Robots and Systems (IROS), 2005.

[8] J. Blanco, W. Burgard, R. Sanz, and J. Fernandez, "Fast face detection for mobile robots by integrating laser range data with vision," in Proc. of the Int. Conf. on Advanced Robotics (ICAR), 2003.

[9] R. Bischoff and V. Graefe, "Hermes - A versatile personal robot assistant," IEEE - Special Issue on Human Interactive Robots for Psychological Enrichment, vol. 92, no. 11, pp. 1759-1779, 2004.

[10] H.-J. Böhme, A. Scheidig, T. Wilhelm, C. Schröter, C. Martin, A. König, S. Müller, and H.-M. Gross, "Progress in the development of an interactive mobile shopping assistant," in Proc. of the Joint Conf. on Robotics: 37th Int. Symp. on Robotics (ISR) and 4th German Conf. on Robotics (Robotik), 2006.

[11] C. Sidner, C. Kidd, C. Lee, and N. Lesh, "Where to look: A study of human-robot engagement," in ACM Int. Conf. on Intelligent User Interfaces (IUI), 2004.

[12] K. Aoyama and H. Shimomura, "Real world speech interaction with a humanoid robot on a layered robot behavior control architecture," in Proc. of IEEE International Conference on Robotics and Automation (ICRA), 2005.

[13] M. Salichs, R. Barber, A. Khamis, M. Malfaz, J.F.Gorostiza, R.Pacheco, R.Rivas, A.Corrales, and E.Delgado, "Maggie: A robotic platform for human-robot social interaction," in IEEE International Conference on Robotics, Automation and Mechatronics (RAM), 2006.

[14] X. Gu and D. Ballard, "An equilibrium point based model unifying movement control in humanoids," in Proc. of Robotics: Science and Systems, 2006.

[15] A. Feldman, "Functional tuning of the nervous system with control of movement or mainenance of a steady posture," Biophysics, vol. 11, pp. 565-587, 1966.

[16] O. Khatib, "Real-time obstacle avoidance for manipulators and mobile robots," Int. Journal of Robotics Research, vol. 5, no. 1, pp. 90-98, 1986.

[17] E. Marchand, A. Rizzo, and F. Chaumette, "Avoiding robot joint limits and kinematic singularities in visual servoing," Proceedings of the 13th IAPR/IEEE Int. Conf. on Pattern Recognition (ICPR'96), vol. A, pp. 297-301, 1996

[18] Y. Nakamura, H. Hanafusa, and T. Yoshikawa, "Task-Priority Based Redundancy Control of Robot Manipulators," The International Journal of Robotics Research, vol. 6, no. 2, pp. 3-15, 1987.

[19] N. Mansard, O. Stasse, F. Chaumette, and K. Yokoi, "Visually-Guided Grasping while Walking on a Humanoid Robot," IEEE International Conference on Robotics and Automation, pp. 3041-3047, 2007.

[20] H. Sugiura, M. Gienger, H. Janssen, and C. Goerick, "Real-Time Self Collision Avoidance for Humanoids by means of Nullspace Criteria and Task Intervals," Proc. of the 6th IEEE-RAS Int. Conf. on Humanoid Robots (Humanoids), pp. 575-580, 2006.

[21] E. Todorov, "Optimality principles in sensorimotor control," Nature Neuroscience, vol. 7, no. 9, pp. 907-915, 2004.

[22] W. Shen and A. R. Galer, "Development of a pressure related assessment model of seating discomfort," Human Factors and Ergonomics Society Annual Meeting Proceedings, Industrial Ergonomics, pp. 831835, 1993.

[23] L. Zhang, "Identifying factors of comfort and discomfort in sitting," Human Factors, vol. 38, no. 3, 1996.

[24] W. Shen and A. M. Vertiz, "Redefining seat comfort," SAE Special Publications, Progress with Human Factors in Automotive Design: Seating Comfort, Visibility, and Safety, vol. 1242, pp. 161-168, 1997.

[25] T. Marler, "A study of multi-objective optimization methods for engineering applications," Masters Thesis, 2005.

[26] S. Behnke, M. Schreiber, J. Stückler, R. Renner, and H. Strasdat, "See, walk, and kick: Humanoid robots start to play soccer," in Proc. of the IEEE-RAS International Conference on Humanoid Robots (Humanoids), 2006, p. 497-503.

[27] H. Kwakernaak and R. Sivan, Linear Optimal Control Systems. Wiley-Interscience, 1972. 